\title{
Efeito agudo do exercício físico aeróbio de diferentes intensidades na proliferação linfocitária de adultos
}

\author{
Acute effect of aerobic exercise training of different intensities on lymphocyte \\ proliferation in adults
}

\section{AUTORES \\ Amanda Archeleiga Guedes ${ }^{1}$ iD \\ Beatriz Augusta Pozzolo ${ }^{1}$ (D) \\ Vanessa Ferrari da Fonseca ${ }^{1}$ (I) \\ Debora Sales Silva Coutinho ${ }^{2}$ (D) \\ Fabiola Iagher ${ }^{2}$ (iD) \\ Anderson Zampier Ulbrich ${ }^{1}$ (ID \\ 1 Universidade Federal do Paraná, Departamento de Medicina Integrada e Grupo de Pesquisa em Medicina do Exercício, Curitiba, Paraná, Brasil. \\ 2 Universidade Federal do Paraná, Departamento de Fisiologia, Curitiba, Paraná, Brasil.}

\section{CONTATO}

Amanda Archeleiga Guedes

archeleiga@gmail.com

medexufpr@gmail.com

Rua Padre Camargo Street, 280, Alto da

Glória, Curitiba, Paraná, Brasil.

CEP: 80060-240.

DOI

10.12820/rbafs.24e0091

\begin{abstract}
RESUMO
O exercício físico gera um desvio da homeostase e a reorganização das respostas de diversos sistemas, entre eles o sistema imune. A resposta aguda do sistema imune pode estar sujeita a mudanças de acordo com a intensidade do exercício praticado. O objetivo do estudo é observar a resposta da proliferação linfocitária à prática de sessões de exercício físico aeróbio de diferentes intensidades. Para tal, foram avaliados dez voluntários (23,50 $\pm 3,43$ anos), que realizaram sessões de exercício aeróbio em bicicleta indoor nas intensidades moderada ( $40-59 \%$ da frequência cardíaca de reserva - FCr) e vigorosa (60-89\% da $\mathrm{FCr}$ ). Houve coleta de sangue venoso para análise da proliferação linfocitária antes (baseline) e após cada uma das sessões. A proliferação linfocitária foi estimulada através de Concanavalina A e mensurada através do ensaio de Alamar Blue ${ }^{\circledR}$ por análise fluorimétrica após $24 \mathrm{~h}, 48 \mathrm{~h}$ e $72 \mathrm{~h}$ de cultivo. A análise estatística compreendeu comparações repetidas entre as diferentes sessões e o baseline por ANOVA two-way, considerando um $\mathrm{p}<0,05$. A sessão de exercício de intensidade vigorosa apresentou diminuição significativa da proliferação linfocitária basal de $21,96 \%(\mathrm{p}=0,011)$ em 24h, 37,46\% ( $p=0,001)$ em $48 \mathrm{~h}$ e $27,61 \%(\mathrm{p}=0,005)$ em $72 \mathrm{~h}$ quando comparada com o baseline. A sessão de exercício de intensidade moderada mostrou um aumento de $42,17 \%$ ( $\mathrm{p}=0,001), 36,89 \%$ $(\mathrm{p}=0,011)$ e $30,84 \%(\mathrm{p}=0,001)$ para os mesmos tempos. Houve diferença significativa no delta de variação na comparação entre as intensidades $(\mathrm{p}<0,005)$. A prática aguda de exercício físico aeróbio em intensidades moderada e vigorosa foi associada ao aumento e diminuição, respectivamente, da proliferação linfocitária de adultos.
\end{abstract}

Palavras-chave: Linfócitos; Exercício físico; Proliferação celular.

\section{ABSTRACT}

Physical exercise causes a deviation in homeostasis and the reorganization of various systems responses, including the immune system. The acute response of the immune system may differ according to the intensity of physical exercise. The objective of this study is to analyze the lymphocyte proliferative response to the practice of aerobic physical exercise sessions of varying intensities. Ten volunteers (23.50 \pm 3.43 years) were evaluated performing aerobic exercise sessions on an indoor bicycle at a moderate intensity (40-59\% of heart rate reserve - HRR) and at a vigorous intensity (60-89\% of HRR). Venous blood was collected for the analysis of lymphocyte proliferation before (baseline) and after each session. Concanavalin A (ConA) was utilized as stimulator and Alamar Blue ${ }^{\circledR}$ assay as measurement. The results were obtained by means of fluorimetric analysis after 24, 48 and 72 hours of cell culture after the exercise sessions. Statistical analysis comprised of repeated comparisons between the different sessions and the baseline by two-way ANOVA, considering $p<0.05$. The vigorous exercise session reported a significant decrease in lymphocyte proliferative response of $21.96 \%(p=0.011)$ in $24 \mathrm{~h}, 37.46 \%(p=0.001)$ in $48 \mathrm{~h}$ and $27.61 \%(p=0.005)$ in $72 \mathrm{~h}$, compared to the baseline. The moderate intensity exercise session reported a significant increase of $42.17 \%(p=0.001)$ in 24h, 36.89\% ( $p=0.011)$ in $48 \mathrm{~h}$ and $30.84 \%(p=0.001)$ in $72 \mathrm{~h}$, compared to the baseline. There was a significant difference in delta variation between the two intensities $(p<0.005)$. Acute aerobic physical exercise at moderate and vigorous intensities caused a decrease and increase, respectively, in the lymphocyte proliferation of young adults.

Keywords: Lymphocyte; Exercise; Cell proliferation.

\section{Introdução}

O exercício físico gera um desvio da homeostase e leva à reorganização das respostas de diversos sistemas, entre eles o sistema imune ${ }^{1}$. Alterações temporárias causadas por uma sessão de exercício são conhecidas como resposta aguda ${ }^{1}$ e ocorrem após a ativação da inflamação localizada, por alterações sistêmicas fisiológicas e comportamentais no organismo ${ }^{2}$.

A resposta aguda é dependente da intensidade e duração do exercício físico ${ }^{2}$. No funcionamento do sis- 
tema imunológico, a resposta se dá em uma curva no formato de " $~ J$, em que exercícios de maior intensidade aumentam a suscetibilidade a doenças infecciosas, especialmente virais de vias aéreas superiores e exercícios de intensidade moderada tem um efeito protetor contra infecções ${ }^{3,4}$. Não há consenso sobre os mecanismos que levam a essa diferença de resposta, mas acredita-se que tenham relação com alterações na funcionalidade de células de defesa ${ }^{2}$.

Os linfócitos estão entre as principais células de defesa do sistema imune, sua capacidade proliferativa, ou seja, capacidade de sofrer expansão monoclonal, é crucial para a resposta imune adaptativa ${ }^{5}$. Sessões de exercício são capazes de afetar a habilidade dos linfócitos de migrarem do sangue para os tecidos periféricos nos locais de inflamação e modificar temporariamente suas funcionalidades ${ }^{2}$.

Green et al. ${ }^{6}$ relatam que o exercício aeróbio sob condições agudas pode alterar a proliferação linfocitária. A capacidade proliferativa linfocitária pode ser usada em contextos clínicos para avaliar a função imune, mas sua resposta ao exercício físico de diferentes intensidades ainda não é bem esclarecida ${ }^{7}$ Estudar essa associação pode fornecer melhores evidências para a prescrição de exercício de forma benéfica e segura ao sistema imune ${ }^{8}$, vislumbrando seu uso como estratégia não farmacológica para aumento da imunidade e da contagem de determinadas populações linfocitárias, bem como uma forma de evitar diminuição no rendimento de atletas e infecções na população em geral ${ }^{2,8}$.

Nesse contexto, o presente artigo objetivou verificar o efeito agudo de diferentes intensidades de exercício físico na proliferação linfocitária de adultos. Hipotetizou-se que o exercício físico aeróbio de intensidade vigorosa e moderada promoveriam diferentes respostas de proliferação linfocitária basal sob estímulo de mitógeno.

\section{Métodos}

Trata-se de um estudo experimental de curta duração, desenvolvido na Unidade Escola de Promoção da Saúde da Universidade Federal do Paraná, Brasil (UE-PS/ UFPR). Este estudo faz parte da segunda fase do projeto de pesquisa intitulado "Comportamento Ativo e Fatores de Risco de Estudantes Universitários: Estudo de Segmento". Todos os sujeitos avaliados na primeira fase do estudo foram convidados a participar de forma espontânea para a fase experimental (intervenção aguda de exercício físico). Foram considerados critérios de exclusão: idade menor que 18 anos ou maior que 35 anos, e ter feito uso de medicamentos anti-inflamatórios ou bebida alcoólica nos dois dias anteriores às intervenções. Foi realizado o cálculo amostral para a fase experimental, sendo utilizado o software $G^{*}$ Power, Versão 3.1.9.4, adotando um poder de $80 \%$ para um teste bicaudal e um erro máximo desejado de $5 \%$. A equação indicou a necessidade de pelo menos seis indivíduos por grupo para obtenção de dados confiáveis de proliferação linfocitária. O estudo foi apreciado pelo Comitê de Ética e Pesquisa do Setor de Ciências da Saúde da UFPR, o qual atende à resolução 466/12 do Conselho Nacional de Saúde, e está de acordo com a Declaração Médica Mundial de Helsinki de 1975 sobre ética em pesquisa médica (CAAE:71645617.4.0000.0102).

Os participantes tiveram sua frequência cardíaca (FC) de repouso aferida e foram submetidos a duas sessões de exercício aeróbio agudo, de diferentes intensidades, com intervalo de sete dias entre as sessões. Foram colhidas amostras de sangue venoso antes e após cada uma das sessões para a determinação da proliferação linfocitária. Foram obtidos dados de nome, idade e sexo dos participantes por questionamento direto, e de nível de atividade física, através de questionário online, para caracterização da amostra. As medidas antropométricas de massa corporal (quilogramas) e estatura (centímetros) foram avaliadas conforme protocolo proposto por Petroski ${ }^{9}$. Com base nessas medidas foi determinado o índice de massa corporal (IMC) $\left(\mathrm{kg} / \mathrm{m}^{2}\right)$ e sua classificação ${ }^{10}$. O Nível de Atividade Física (NAF) foi avaliado por meio do Questionário Internacional de Atividade Física (IPAQ) versão curta e os participantes foram classificados em sedentários, levemente, moderadamente e muito ativos ${ }^{11}$.

Os participantes realizaram duas sessões de exercícios físicos aeróbios contínuos de intensidades diferentes, com intervalo de sete dias entre elas. Foi solicitado aos participantes que não realizassem exercícios físicos nas $24 \mathrm{~h}$ que antecederam as intervenções propostas. As sessões foram realizadas em bicicleta indoor (Schwinn ${ }^{\circledast}$, AC Sport, USA) e tiveram duração aproximada de 40 minutos, sendo os 10 minutos iniciais de aquecimento em intensidade leve, 30 minutos em intensidade específica, moderada durante a primeira sessão e vigorosa durante a segunda, finalizando com cinco minutos de recuperação em intensidade leve. A velocidade em rotações por minuto na bicicleta foi mantida entre 70 a $80 \mathrm{rpm}$ de forma constante. Os Watts foram verificados durante as intervenções e são derivados da cadência e resistência imposta na bicicleta utilizada, sendo a carga 
ajustada para que o participante se mantivesse nas zonas de frequência cardíacas propostas.

As intensidades do exercício aeróbio foram calculadas previamente pela estimativa da frequência cardíaca de reserva $(\mathrm{FCr})$, mediante o protocolo de cálculo proposto pelo American College of Sports $\mathrm{Me}$ dicine $(\mathrm{ACSM})^{12}$. Para mensuração da $\mathrm{FC}$ em repouso (FCrepouso) foi utilizado o monitor cardíaco (Polar ${ }^{\circledR}$ FT1, Finlândia) e o participante permaneceu em ambiente silencioso e climatizado na posição deitada em decúbito dorsal sobre uma maca confortável, pelo tempo de 10 minutos, durante período vespertino. A FC máxima (FCmax) foi estimada através da fórmula proposta por Astrand ${ }^{13}$. Os valores de FC foram classificados de acordo com a recomendação do $\mathrm{ACSM}^{12}$, considerando exercício aeróbio de intensidade leve aquele que se atinge $30-39 \%$ da $\mathrm{FCr}$, intensidade moderada entre 40-59\% da FCr e intensidade vigorosa o exercício que atinge entre $60-89 \%$ da $\mathrm{FCr}^{12}$.

Durante as sessões de exercício a FC dos participantes foi controlada por monitor cardíaco (Polar ${ }^{\circledR}$ FT1, Finlândia), a pressão arterial sanguínea aferida por meio dos equipamentos de esfigmomanômetro aneroide (Welch Allyn ${ }^{\circledast}$, Durashock DS44-BR, USA) e estetoscópio (Littmann ${ }^{\circledast}$, Classic II, USA), em intervalos de 10 minutos, conjuntamente com a percepção de esforço muscular e respiratória pela escala de Borg adaptada ${ }^{14}$.

Para determinação da resposta proliferativa linfocitária foram colhidas amostras de sangue venoso dos participantes antes e após cada uma das sessões de exercícios, utilizando tubos de ensaio de 7,5ml com EDTA (Sarstedt ${ }^{\varpi}$, Monovettes AG, Alemanha), para determinação da proliferação linfocitária basal (baseline) e das proliferações após as sessões de intensidade específica. Alíquotas de $4 \mathrm{ml}$ da amostra foram transferidas para tubos cônicos de polipropileno estéril contendo $4 \mathrm{ml}$ de Histopaque-1077 (Sigma ${ }^{\circledR}$, Histopaque-1077, USA). Após centrifugação a $400 \times \mathrm{g}$ por 35 minutos, a nuvem linfocitária formada foi aspirada e lavada com solução hemolítica (4.01g NH4Cl; 0.8g EDTA-Na; 0.42g Na2CO3; qsp.500 $\mathrm{mL}_{2} \mathrm{O}$ ) para hemólise de eritrócitos residuais. As células foram lavadas com PBS (phosphate buffered saline) e o conteúdo foi centrifugado a $2000 \mathrm{x} g$ por $5 \mathrm{mim}$ três vezes. $\mathrm{O}$ botão celular resultante foi ressuspendido em $4 \mathrm{~mL}$ de RPMI-1640 (Sigma ${ }^{\circledR}$, Medium RPMI 1640, USA). A concentração celular foi determinada por contagem em câmara de Neubauer e a viabilidade celular pelo método de exclusão com azul Tripan. A amostra foi diluída em meio RPMI-1640 para obtenção da concentração celular final necessária para o cultivo.

Os linfócitos dos diferentes grupos foram cultivados em placas com fundo chato preto de 96 poços em meio de cultura RPMI-1640 enriquecido com 10\% de soro fetal bovino e contendo penicilina 10.000UI/ estreptomicina $10 \mathrm{mg} / \mathrm{L}$. Cada poço continha um volume de $160 \mu \mathrm{L}$ de solução contendo $5 \times 10^{4}$ células/ escavação, e mais: $20 \mu \mathrm{L}$ /poço do meio RPMI-1640 ou $20 \mu \mathrm{L}$ /poço do mitógeno Concanavalina A (ConA). Então foram adicionados $20 \mu \mathrm{L} /$ escavação de Alamar Blue (Thermo Fisher ${ }^{\infty}$, alamarBlue-DAL1025, USA) e as placas foram mantidas a $37^{\circ} \mathrm{C}$ em atmosfera de $95 \%$ ar e $5 \%$ de $\mathrm{CO}_{2}$ por 72 horas em incubadora de cultivo (ShelLab ${ }^{\circledR}$ 2350T, USA). A determinação da porcentagem de redução do Alamar Blue foi realizada por leitura fluorimétrica, com comprimento de onda de excitação entre 530-560nm e comprimento de onda de emissão em 590nm pelo leitor multifuncional de microplacas (Tecan ${ }^{\oplus}$ Infinite M200, USA) após 24, 48 e 72 horas do plaqueamento. Todas as análises foram realizadas em duplicata.

A análise estatística foi iniciada por meio da normalidade dos dados (Shapiro Wilk), seguida de análise descritiva de medidas de tendência central (média e desvio padrão) e frequência (\%) na caracterização da amostra. Para verificar o efeito das sessões e as variáveis hemodinâmicas, de percepção de esforço e fisiológicas, foi realizada análise de variância de duas vias para medidas repetidas (ANOVA two-way). Todas as análises foram realizadas no $\mathrm{SPSS}^{\circledast}$ para Windows ${ }^{\circledR}$, versão 20.0, sendo considerado relevante um $\mathrm{p}<0,05$ e os gráficos plotados no software $\mathrm{GraphPad}^{\oplus}$ Prisma versão 6.01 para Windows ${ }^{\circledR}$.

\section{Resultados}

Foram convidados a participar da pesquisa 79 adultos de ambos os sexos participantes da primeira fase do projeto e apenas 10 aceitaram participar da fase experimental. A principal causa de recusa foi a indisponibilidade para realizar as sessões de exercícios nos dias e horários propostos. As características antropométricas e de NAF da amostra $(n=10)$ estão descritas na Tabela 1. Com base no recrutamento proposto no estudo, a maior prevalência foi do sexo feminino, e não houve perdas amostrais durante a execução do estudo. Observou-se pela classificação do $\mathrm{IMC}^{8}$ que todos se encontravam dentro da faixa de peso normal. Com relação ao NAF, todos os sujeitos de estudo foram classificados como moderadamente ativos? 
Tabela 1 - Características descritivas dos participantes da pesquisa $(n=10)$.

\begin{tabular}{lc}
\hline Características & Média \pm Desvio Padrão \\
\hline Idade (anos) & $23,50 \pm 3,43$ \\
Mulheres, $\mathrm{n}(\%)$ & $07(70 \%)$ \\
Massa Corporal $(\mathrm{kg})$ & $62,50 \pm 9,92$ \\
Estatura $(\mathrm{cm})$ & $168,00 \pm 8,11$ \\
IMC $\left(\mathrm{kg} / \mathrm{m}^{2}\right)$ & $22,05 \pm 3,43$ \\
Minutos por semana de AF $(\mathrm{min})$ & $90,50 \pm 24,09$ \\
METs $(\mathrm{min} / \mathrm{sem})$ & $1250,30 \pm 584,08$ \\
\hline
\end{tabular}

$\mathrm{IMC}=$ índice de massa corporal; $\mathrm{AF}=$ atividade física; $\mathrm{METs}=$ equivalentes metabólicos.
As variáveis coletadas durante as sessões de exercício como FC atingidas, pressão arterial, potência em Watts da bicicleta e percepção subjetiva de esforço muscular e respiratório, estão reportadas na Figura 1. $\mathrm{Na}$ variável FC encontramos diferença significativa entre os valores atingidos nos tempos de início, exercício e recuperação. Houve diferença significativa entre as médias de FC atingidas nas duas sessões, sendo maiores no exercício vigoroso, todos os participantes se encontraram nas faixas de FC propostas durante as sessões.
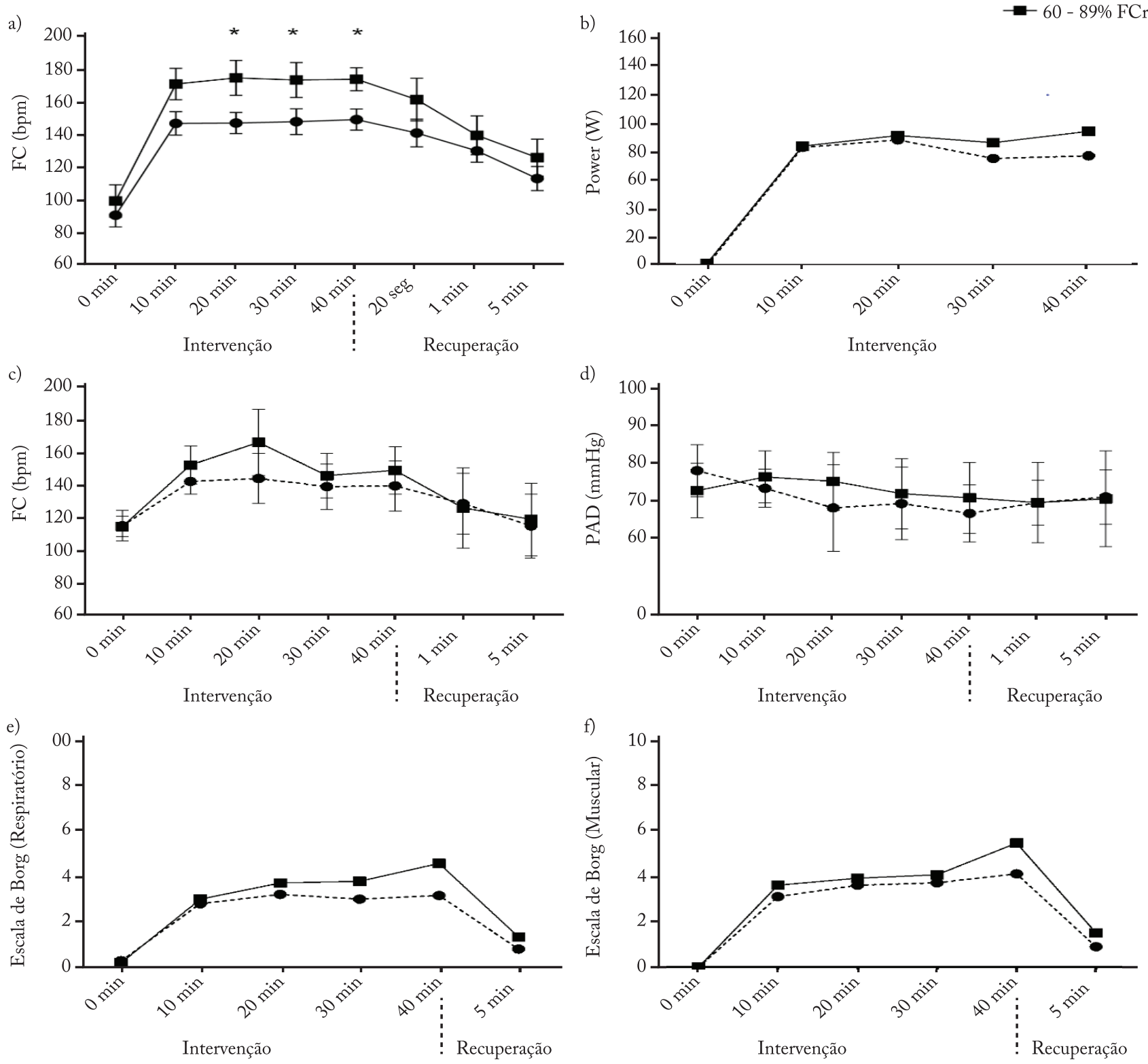

Figura 1 - Efeitos da intensidade do exercício físico nas respostas hemodinâmicas e nas escalas auto avaliativas do esforço durante as intervenções Valores representados por médias. (A) Média das frequências cardíacas; (B) Média das potências em Watts; (C) e (D) Médias da pressão arterial sistólica e diastólica durante as sessões; (E) e (F) Escala de percepção de esforço respiratório e muscular de Borg. * p $<0,05$ na comparação com o baseline e entre as intensidades. $\mathrm{FC}=$ frequência cardíaca; $\mathrm{FCr}=$ frequência cardíaca de reserva; $\mathrm{PAS}=$ pressão arterial sistólica, $\mathrm{PAD}=$ pressão arterial diastólica. 


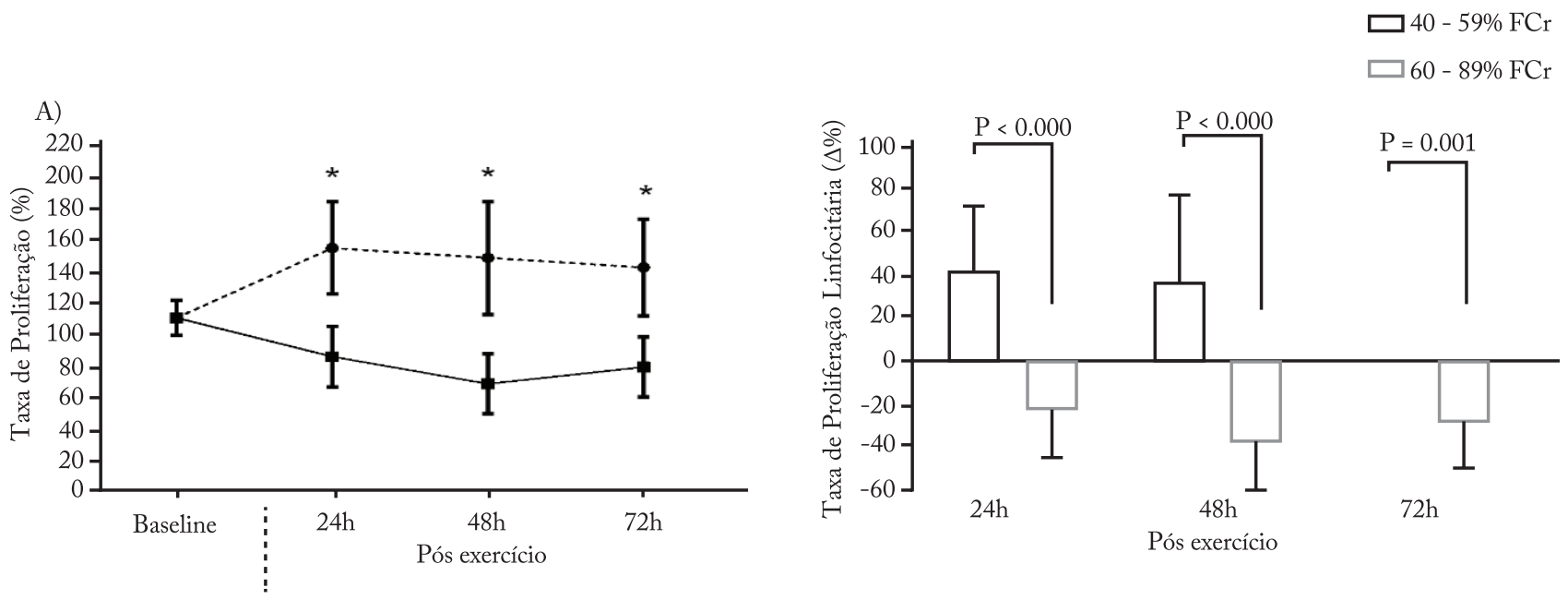

Figura 2 - Resposta proliferativa linfocitária as diferentes intensidades de exercício físico.

(A) Proliferação linfocitária absoluta em termos de porcentagem de redução do Alamar Blue ${ }^{\circledR}$, nos tempos baseline, 24h, 48h e $72 \mathrm{~h}$ após as sessões de exercício físico. (B) Delta da variação percentual da proliferação linfocitária basal após as sessões de intensidade moderada e vigorosa. ${ }^{*} \mathrm{p}<0,05$ na comparação com o baseline e entre as intensidades. $\mathrm{AB}=$ Alamar $\mathrm{Blue} ; \mathrm{FC}=$ frequência cardíaca de reserva.

Não houve diferença significativa nos valores da pressão arterial diastólica (PAD), pressão arterial sistólica (PAS) e nas escalas subjetivas de Borg de esforço na comparação entre as sessões de exercício físico.

A Figura 2 apresenta a proliferação linfocitária. Não houve diferença significativa na proliferação nos tempos de $24 \mathrm{~h}, 48 \mathrm{~h}$ e $72 \mathrm{~h}$ quando comparados na mesma sessão. Pelo delta de variação da proliferação linfocitária, a sessão de exercício de intensidade moderada mostrou um aumento significativo de proliferação de $42,2 \%(\mathrm{p}=0,001), 36,9 \%(\mathrm{p}=0,011)$ e $30,8 \%(\mathrm{p}$ $=0,01)$ para os tempos $24 \mathrm{~h}, 48 \mathrm{~h}$ e 72 horas, respectivamente, quando comparada com a proliferação basal. $\mathrm{Na}$ sessão de exercício vigoroso houve uma diminuição significativa de $21,9 \%$ ( $\mathrm{p}=0,011), 37,5 \%(\mathrm{p}=0,01)$ e $27,6 \%(p=0,005)$ na proliferação linfocitária basal. $\mathrm{Na}$ comparação entre as duas intensidades, há uma diferença percentual de proliferação de $64,1 \% \mathrm{em} 24 \mathrm{~h}(\mathrm{p}=$ $0,0001), 74,3 \%$ em $48 \mathrm{~h}(\mathrm{p}=0,0001)$ e $58,5 \%$ em $72 \mathrm{~h}$ $(\mathrm{p}=0,002)$ sendo que na sessão de intensidade vigorosa houve menor proliferação linfocitária que na sessão de atividade moderada.

\section{Discussão}

No presente estudo, investigou-se como o exercício físico de intensidades moderada e vigorosa afetam agudamente a capacidade proliferativa linfocitária de adultos. Após uma sessão de intensidade vigorosa foi observada uma diminuição significativa na resposta proliferativa, quando comparada à resposta basal e ao exercício de intensidade moderada. Já na sessão de exercício moderado, sob as mesmas condições, observou-se um aumento significativo na proliferação dos linfócitos. Estes dados sugerem que a resposta proliferativa ao exercício agudo é dependente da intensidade em que ele é realizado.

O perfil de resposta encontrado é correspondente à curva de resposta imune ao exercício físico ${ }^{3}$, sendo que a resposta proliferativa linfocitária pode ser um dos responsáveis pelo formato de " $\rho$ " da curva, com piora na função imune em intensidade mais elevadas ${ }^{2,3}$. Isso é corroborado pela descrição de maior suscetibilidade a infecções virais após exercícios extenuantes ${ }^{15}$ como maratonas e triatlos ${ }^{16-18}$ já que os linfócitos, tem um papel central na resposta imune a antígenos, especialmente virais ${ }^{4}$.

Conforme meta-análise, a diminuição da proliferação linfocitária após a prática de exercício de alta intensidade é bem documentada ${ }^{7}$. Os possíveis mecanismos envolvidos são a ação da epinefrina e do cortisol ${ }^{19}$. Pela atividade estressora do exercício e a lesão muscular provocada, há um aumento dos níveis de epinefrina e cortisol no organismo ${ }^{2}$. A epinefrina tem ação sobre receptores beta-adrenérgicos, diminuindo a secreção de interleucina 2 (IL-2) e a expressão de receptores de IL-2, os quais são fundamentais para a expansão e crescimento das populações linfocitárias ${ }^{20}$. O cortisol também parece inibir a proliferação por ação direta na célula linfocitária e por inibição de IL-2 ${ }^{19-21}$. Além disso, pode haver um mecanismo adicional decorrente da ação da epinefrina e do cortisol sobre os monócitos, que diminuiria a expressão do complexo major de histocompatibilidade $(\mathrm{CMH})$ de classe II e sua atuação 
como célula apresentadora de antígeno, bem como sua incitação à proliferação linfocitária ${ }^{22}$.

O aumento da proliferação linfocitária após exercícios leves e moderados também é documentado em pesquisas com modelos animais e envolvendo indivíduos saudáveis ${ }^{23-25}$, porém os mecanismos que levariam a esse aumento ainda não estão bem esclarecidos ${ }^{24}$. Acredita-se que tenha relação com a menor dose de cortisol e epinefrina gerados, com o aumento da produção de IL-4 e alterações no metabolismo de glucose e glutamina de linfócitos $\mathrm{T}^{25}$.

Como limitações temos a disparidade entre homens e mulheres, uma vez que estas voluntariamente se dispuseram a participar. A alta suscetibilidade da célula linfocitária a pequenas mudanças no perfil inflamatório do indivíduo que não puderam ser excluídas. $\mathrm{O}$ não controle do consumo de alimentos, hidratação e consumo de cafeína dos participantes, bem como doenças crônicas e sazonais não relatadas ou pouco evidentes. Ademais, destacamos a limitação quanto à fórmula utilizada para estimar as zonas de treinamento.

Sumarizando, o presente estudo encontrou que há uma associação entre a intensidade do exercício e proliferação linfocitária. Em exercício de maior intensidade houve a diminuição da resposta proliferativa linfocitária, enquanto exercícios de intensidade moderada cursaram com um aumento da resposta proliferativa de adultos. A partir dos resultados encontrados, a prática de exercícios vigorosos pode ter um efeito deletério transitório para o funcionamento do sistema imune e gerar uma propensão a infecções. Já a intensidade moderada pode se mostrar benéfica ao sistema imune, com aumento aproximado de $35 \%$ da proliferação linfocitária basal, sugerindo um benefício maior na prática e prescrição de tal intensidade.

\section{Conflito de interesse}

Os autores declaram não haver conflito de interesses.

\section{Contribuição dos autores}

Guedes AA, Pozollo BA, Fonseca VF, Iagher F e Ulbrich AZ desenharam a pesquisa. Guedes AA, Pozollo BA, Fonseca VF e Coutinho DSS realizaram experimentos. Guedes AA, Coutinho DSS, Iagher F e Ulbrich AZ analisaram os dados. Guedes AA, Pozollo BA, Fonseca VF, Coutinho DSS, Iagher F e Ulbrich AZ interpretaram resultados de experimentos; Guedes AA, Coutinho DSS, Iagher F e Ulbrich AZ elaboraram as figuras e tabelas do manuscrito. Guedes AA, Pozollo BA, Fonseca VF, Coutinho DSS, Iagher F e Ulbrich AZ editaram, revisaram e aprovaram a versão final do manuscrito e concordam em prestar contas de todos os aspectos do trabalho, garantindo que as questões relacionadas à exatidão ou à integridade de qualquer parte do trabalho sejam adequadamente investigadas e resolvidas. Todas as pessoas designadas como autores se qualificam para a autoria, e todos aqueles que se qualificam para autoria são listados.

\section{Agradecimentos}

Os autores agradecem aos alunos da professora Fabiola Iagher do Laboratório de Fisiologia da Universidade Federal do Paraná e aos demais participantes do Grupo de Pesquisa em Medicina do Exercício (MedEx) que tanto colaboraram para a realização das análises.

\section{Referências}

1. Costa Rosa LFPB, Vaisberg MW. Influências do exercício na resposta imune. Rev Bras Med do Esporte. 2002;8:167-72.

2. Gleeson M. Immune function in sport and exercise. J Appl Physiol. 2007;103(2):693-9.

3. Nieman DC. Exercise, infection, and immunity. Int J Sports Med. 1994;15 Suppl 3:S131-41.

4. Martin SA, Pence BD, Woods JA. Exercise and respiratory tract viral infections. Exerc Sport Sci Rev. 2009;37(4):157-64.

5. Kindt T, Goldsby R, Osborne B. Kuby immunology. New York: W.H. Freeman and Company; 2007.

6. Green KJ, Rowbottom DG, Mackinnon LT. Exercise and T-lymphocyte function: a comparison of proliferation in PBMC and NK cell-depleted PBMC culture. J Appl Physiol. 2002;92(6):2390-5.

7. Siedlik JA, Benedict SH, Landes EJ, Weir JP, Vardiman JP, Gallagher PM. Acute bouts of exercise induce a suppressive effect on lymphocyte proliferation in human subjects: A meta-analysis. Brain Behav Immun. 2016;56:343-51.

8. Eidam CL, Lopes ASL, Oliveira OVO. Prescrição de exercícios físicos para portadores do vírus HIV. Rev Bras Ciência e Mov. 2008;13(3):81-8.

9. Peroski EL. Antropometria: técnica e padronizações. 4th ed. Porto Alegre; 2019.

10. Obesity N, Initiative E. Guide Identification , Evaluation, and Treatment of Overweight and Obesity in Adults. Obesity. 2000

11. Matsudo S, Araújo T, Matsudo V, Andrade D, Andrade E. Questionário internacional de atividade física (IPAQ): estudo de validade e reprodutibilidade no Brasil. Rev Bras Ativ Fis Saúde. 2001;6(2):05-18.

12. Heath EH. ACSM's Guidelines for Exercise Testing and Prescription, 9th Edition. Med Sci Sport Exerc. 2014.

13. Astrand P. Experimental studies of physical working capacity in relation to sex and age. Copenhagen: 1VIunksgaard. 1952.

14. Borg G. Borg's Perceived Exertion And Pain Scales. Human Kinetics. 1998.

15. Friman G, Ilback NG. Acute infection: metabolic responses, effects on performance, interaction with exercise, and myocarditis. Int J Sports Med. 1998;19 Suppl 3:S172-82.

16. Gundacker ND, Rolfe RJ, Rodriguez JM. Infections associated with adventure travel: A systematic review. Travel Med Infect Dis. 2017;16:3-10.

17. Bassit RA, Sawada LA, Bacurau RF, Navarro F, Costa Rosa LF. The effect of BCAA supplementation upon the immune response of triathletes. Med Sci Sports Exerc. 2000;32(7):1214-9. 
18. Henson DA, Nieman DC, Pistilli EE, Schilling B, Colacino A, Utter AC, et al. Influence of carbohydrate and age on lymphocyte function following a marathon. Int J Sport Nutr Exerc Metab. 2004;14(3):308-22.

19. Pedersen BK, Toft AD. Effects of exercise on lymphocytes and cytokines. Br J Sports Med. 2000;34(4):246-51.

20. Zalli A, Bosch JA, Goodyear O, Riddell N, McGettrick HM, Moss $\mathrm{P}$, et al. Targeting ss 2 adrenergic receptors regulate human $\mathrm{T}$ cell function directly and indirectly. Brain Behav Immun. 2015;45:211-8.

21. Kruger K, Agnischock S, Lechte rmann A, Tiwari S, Mishra $\mathrm{M}$, Pilat C, et al. Intensive resistance exercise induces lymphocyte apoptosis via cortisol and glucocorticoid receptordependent pathways. J Appl Physiol. 2011;110(5):1226-32.

22. Rosa LF. Effect of adrenaline on lymphocyte metabolism and function. A mechanism involving cAMP and hydrogen peroxide. Cell Biochem Funct. 1997;15(2):103-12.
23. Yeh S-H, Lai H-L, Hsiao C-Y, Lin L-W, Chuang Y-K, Yang $\mathrm{Y}-\mathrm{Y}$, et al. Moderate physical activity of music aerobic exercise increases lymphocyte counts, specific subsets, and differentiation. J Phys Act Health. 2014;11(7):1386-92.

24. Rhind SG, Shek PN, Shinkai S, Shephard RJ. Effects of moderate endurance exercise and training on in vitro lymphocyte proliferation, interleukin-2 (IL-2) production, and IL-2 receptor expression. Eur J Appl Physiol Occup Physiol. 1996;74(4):348-60.

25. Navarro F, Bacurau AVN, Pereira GB, Araujo RC, Almeida SS, Moraes MR, et al. Moderate exercise increases the metabolism and immune function of lymphocytes in rats. Eur J Appl Physiol. 2013;113(5):1343-52.

Recebido: 23/08/2019 Aprovado: 03/12/2019

\section{Como citar este artigo:}

Guedes AA, Pozzolo BA, Fonseca VF, Coutinho DSS, Iagher F, Ulbrich AZ. Efeito agudo do exercício físico aeróbio de diferentes intensidades na proliferação linfocitária de adultos. Rev Bras Ativ Fis Saúde. 2019;24:e0091. DOI: 10.12820/rbafs.24e0091 